\title{
ON THE SCHUR-BAER PROPERTY
}

\author{
MOHAMMAD REZA R. MOGHADDAM
}

(Received 27 June 1980)

Communicated by D. E. Taylor

\begin{abstract}
In 1957 P. Hall conjectured that every (finitely based) variety has the property that, for every group $G$, if the marginal factor-group is finite, then the verbal subgroup is also finite. The content of this paper is to present a precise bound for the order of the verbal subgroup of a group $G$ when the marginal factor-group is of order $p^{n}$ ( $p$ a prime and $n>1$ ) with respect to the variety of polynilpotent groups of a given class row. We also construct an example to show that the bound is attained and furthermore, we obtain a bound for the order of the Baer-invariant of a finite $p$-group with respect to the variety of polynilpotent groups.
\end{abstract}

1980 Mathematics subject classification (Amer. Math. Soc.): 20 E 10, 20 F 12.

\section{Introduction}

We assume that the reader is familiar with the notions of the verbal subgroup, $V(G)$, and the marginal subgroup, $V^{*}(G)$, associated with a variety of groups, $\mathbf{V}$, and a group $G$ (see P. Hall (1957)).

Let $F_{\infty}$ be the group freely generated by an infinite countable set $X=$ $\left\{x_{1}, x_{2}, \ldots, x_{i}, \ldots\right\}$. Let $v=v\left(x_{i}, \ldots, x_{i}\right)$ be a reduced word in $F_{\infty}$. The word $v$ has the Schur-Baer Property, if for any group $G$ for which the marginal factor-group, $G / V^{*}(G)$, is finite, then the verbal subgroup, $v(G)$, is also finite (P. Hall (1957)). Similarly, a variety $\mathbf{V}$ is called a Schur-Baer Variety if it carries the above property.

P. Hall in 1957 conjectured that every word has the Schur-Baer property. It is an unsettled problem whether all words are Schur-Baer words. For a survey of 
present knowledge on this problem the reader is referred to D. Robinson (1972). No-one, in fact, has tried to construct any suitable bound for the order of the verbal subgroup in terms of the order of the given marginal factor-group. However, when the central factor-group of a group $G$ is of order $p^{n}, p$ a prime number, J. Wiegold (1965) has given a precise bound for the order of the derived group $G^{\prime}$. He also uses this to derive a bound for the Schur-multiplicator of a finite group of order $p^{n}$, which was first obtained by J. A. Green (1956).

The main purpose of this paper is to give a precise bound for the order of the verbal subgroup of a group $G$ in the case that the marginal factor-group is of order $p^{n}$. In fact, it is shown that if $\mathbf{V}$ is the variety of polynilpotent groups of a given class row, and $G$ is a group with marginal factor-group, $G / V^{*}(G)$, of order $p^{n}$ then

$$
|V(G)|<\left|V M\left(Z_{p}^{n}\right)\right|,
$$

where $Z_{p}^{n}$ denotes the direct product of $n$-copies of the cyclic group of order $p$, and $\operatorname{VM}(G)$, for any group $G$, is the Baer-invariant of the group $G$ with respect to the variety $\mathbf{V}$ (see Section 2, for the definition).

If a variety $\mathbf{V}$ satisfies this property, then we shall call $\mathbf{V}$ a $p^{n}$-Schur-Baer variety (henceforth $p^{n}$-S.B. variety). Then the above bound is used to obtain a bound for the corresponding Baer-invariant of a finite group.

\section{Notation and preliminaries}

If $x$ and $y$ are elements of a group $G$ then $[x, y]$, the commutator of $x$ and $y$, and $x^{y}$ denote the elements $x^{-1} y^{-1} x y$ and $y^{-1} x y$, respectively. The higher commutators are defined inductively, that is,

$$
\left[x_{1}, x_{2}, \ldots, x_{n}\right]=\left[\left[x_{1}, x_{2}, \ldots, x_{n-1}\right], x_{n}\right] .
$$

If $H$ and $K$ are subgroups of a group $G$ then $[H, K]$ denotes the subgroup of $G$ generated by all the commutators $[h, k]$ with $h$ in $H$ and $k$ in $K$; in particular, the derived group of $G$ is $[G, G]$ which is denoted by $G^{\prime}$. The lower central series of $G$ is denoted by

$$
G=\gamma_{1}(G) \supseteq G^{\prime}=\gamma_{2}(G) \supseteq \gamma_{3}(G) \supseteq \ldots
$$

For our convenience we denote the commutator $[x, y, \ldots, y](n$ copies of $y)$ by $\left[x,{ }_{n} y\right]$ and similarly, the commutator subgroup $[H, K, \ldots, K](n$ copies of $K)$ will be denoted by $\left[H,{ }_{n} K\right]$.

Outer commutators are defined inductively as follows: The word $x_{i}$ is an outer commutator word (henceforth o.c. word) of weight one. If $u=u\left(x_{1}, \ldots, x_{s}\right)$ and 
$v=v\left(x_{s+1}, \ldots, x_{s+t}\right)$ are o.c. words of weights $s$ and $t$ respectively, then

$$
w\left(x_{1}, \ldots, x_{s+t}\right)=\left[u\left(x_{1}, \ldots, x_{s}\right), v\left(x_{s+1}, \ldots, x_{s+t}\right)\right]
$$

is an o.c. word of weight $s+t$. We shall write $w=[u, v]$, and plainly for any group $G$ we have $w(G)=[u(G), v(G)]$.

Any word $v$ in $F_{\infty}^{\prime}$ is called a commutator word. Clearly for any group $G$, if $v$ is a commutator word then $v(G) \subset G^{\prime}$. Hence the variety $\mathbf{V}$ contains the variety of abelian groups $\mathbf{A}$, if $V(G) \subset G^{\prime}$.

The reader is referred to M. Hall (1959), p. 166; for the definition of basic commutators.

If $S$ is a subset of a group $G$, then $\langle S\rangle$ denotes the group generated by $S$ and $\langle\bar{S}\rangle$ is the normal closure of $\langle S\rangle$. Finally, $|G|$ denotes the order of the finite group $G$.

Let $\mathbf{V}$ be a variety of group defined by the set of laws $V$, and let $G$ be a group with a free presentation $1 \rightarrow R \rightarrow F \rightarrow G \rightarrow 1$, where $F$ is a free group. Then the Baer-invariant of $G$, with respect to the variety $V$, denoted by $V M(G)$, is defined to be

$$
\frac{R \cap V(F)}{\left[R V^{*} F\right]},
$$

where $V(F)$ is the verbal subgroup of $F$ and $\left[R V^{*} F\right]$ is the least normal subgroup of $F$ contained in $R$ (see Lemma 1.2(ii)) generated by the following set;

$$
\begin{aligned}
& \left\{v\left(f_{1}, \ldots, f_{i-1}, f_{i} r, \ldots, f_{s}\right)\left(v\left(f_{1}, \ldots, f_{s}\right)\right)^{-1}\right. \\
& \left.\quad r \in R, f_{i} \in F, 1 \leqslant i \leqslant s, v \in V\right\} .
\end{aligned}
$$

(See C. R. Leedham-Green and S. McKay (1976), from where we take our notation, and H. Neumann (1967) for the notion of varieties of groups.)

One can see that the Baer-invariant of the group $G$ is always abelian and independent of the choice of the presentation of $G$ (see M. R. R. Moghaddam (1975) or Leedham-Green and McKay (1976)).

In particular, if $\mathbf{V}$ is the variety of abelian or nilpotent groups of class at most $c(c>1)$, then the Baer-invariant of the group $G$ will be $R \cap F^{\prime} /[R, F]$, which by I. Schur (1907) is isomorphic to the multiplicator of $G$ (denoted by $M(G)$ ), or $R \cap \gamma_{c+1}(F) /\left[R,{ }_{c} F\right]$, respectively (see Moghaddam (1979)).

The following lemma of commutator calculus will be needed later. 
LEMMA 2.1. Let $F$ be a free group freely generated by the set $\left\{x_{1}, \ldots, x_{n}\right\}$. Then for any integers $c>0$ and $m>0$,

(i) $\left[x_{i_{1}}^{m}, x_{i_{2}}, \ldots, x_{i_{c+1}}\right]$

$$
\equiv\left[x_{i_{1}}, \ldots, x_{i_{c+1}}\right]^{m}\left[x_{i_{1}}, x_{i_{2}}, x_{i_{1}}, x_{i_{3}}, \ldots, x_{i_{c+1}}\right]^{\left(\frac{m}{2}\right)} \bmod \gamma_{c+3}(F),
$$

(ii) $\left[x_{i}, \ldots, x_{i_{j-1}}, x_{i_{j}}^{m}, x_{i_{j+1}}, \ldots, x_{i_{i+1}}\right]$

$$
\equiv\left[x_{i}, \ldots, x_{i_{c+1}}\right]^{m}\left[x_{i_{1}}, \ldots, x_{i_{j-1}}, x_{i}, x_{i j}, x_{i_{j+1}}, \ldots, x_{i_{c+1}}\right] \bmod \gamma_{c+3}(F),
$$

for all $j=2, \ldots, c+1$, where $\left(\begin{array}{l}m \\ 2\end{array}\right)=m(m-1) / 2$.

The following lemma is due to P. Hall (1957) which gives the relation between the verbal and the marginal subgroups of a group.

Lemma 2.2. Let $\mathrm{V}$ be a variety of groups and $G$ be any group. Then

(i) $V\left(V^{*}(G)\right)=\langle 1\rangle$. In particular, $V(G)=\langle 1\rangle$ if and only if $G=V^{*}(G)$.

(ii) If $K / V^{*}(G)$ is in the centre of $G / V^{*}(G)$, then $[K, V(G)]=1$. In particular $\left[V^{*}(G), V(G)\right]=1$.

The following lemma is also needed and its proof is straightforward.

LeMMA 2.3. Let $\mathrm{V}$ be a variety of groups and $G$ be a group with a normal subgroup $N$. Then

(i) $V(G / N)=V(G) N / N$,

(ii) $\left[N V^{*} G\right]$ is the least normal subgroup of $G$ contained in $N$ such that $N /\left[N V^{*} G\right] \subset V^{*}\left(G /\left[N V^{*} G\right]\right)$,

(iii) $[N, V(G)] \subset\left[N V^{*} G\right] \subset N \cap V(G)$.

We remark that if $\mathbf{V}$ is the variety of groups defined by o.c. words $V$, then for any group $G$

$$
\begin{aligned}
V^{*}(G)=\{a \in G ; & \left(g_{1}, \ldots, g_{i-1}, a, g_{i+1}, \ldots, g_{s}\right)=1, \\
1 & \left.\leqslant i \leqslant s, g_{1}, \ldots, g_{s} \in G \text { and for all } v \text { in } V\right\} .
\end{aligned}
$$

This is the content of R. F. Turner-Smith (1964), Theorem 2.3. Hence, if $N$ is a normal subgroup of $G$ then

$$
\begin{aligned}
{\left[N V^{*} G\right]=\left\langle v\left(g_{1}, \ldots, g_{i-1}, b, g_{i+1}, \ldots, g_{s}\right)\right.} & \\
& 1<i\left\langle s, g_{1}, \ldots, g_{s} \in G, \text { and } b \in N\right\rangle .
\end{aligned}
$$

These facts will be used without further reference. 
Defintion 2.4. Let $v\left(x_{1}, \ldots, x_{s}\right)$ and $u\left(x_{1}, \ldots, x_{t}\right)$ be two words. Then the composite of $v$ and $u, v \circ u$, is defined as follows:

$$
v \circ u=v\left(u\left(x_{1}, \ldots, x_{t}\right), \ldots, u\left(x_{(s-1) t+1}, \ldots, x_{s t}\right)\right) \text {. }
$$

The following lemma of J. A. Hulse and J. C. Lennox (1976) relates the verbal and the marginal subgroups of the composite of two words to its components.

LEMMA 2.5. Let $v=v\left(x_{1}, \ldots, x_{s}\right)$ be an o.c. word and $u=u\left(x_{1}, \ldots, x_{t}\right)$ be any other word. Then for every group $G$

(i) $v \circ u(G)=v(u(G))$,

(ii) $(v \circ u)^{*}(G)=H$, where $H / v^{*}(u(G))=u^{*}\left(G / v^{*}(u(G))\right)$.

Proof. (i) By induction on the weight of $v$ (henceforth wt. $v$ ). Suppose $v$ is of weight two, then by Definition 2.4 ,

$$
\begin{aligned}
v \circ u(G) & =\left\langle\left[u\left(g_{1}, \ldots, g_{t}\right), u\left(g_{t+1}, \ldots, g_{2 t}\right)\right] ; g_{i} \in G, 1<i\langle 2 t\rangle\right. \\
& =[u, u](G),
\end{aligned}
$$

and $v(u(G))=\left\langle\left[h_{1}, h_{2}\right] ; h_{j} \in u(G), j=1,2\right\rangle=[u(G), u(G)]$. Hence the equality holds, when the word $v$ is of weight two.

Assume the result holds for all o.c. words of weight less than $n$, and let $v=\left[v_{1}, v_{2}\right]$, where wt. $v=$ wt. $v_{1}+$ wt. $v_{2}=n$, then

$$
v(u(G))=\left[v_{1}(u(G)), v_{2}(u(G))\right] .
$$

By induction hypothesis, $\nu_{i} \circ u(G)=v_{i}(u(G))$, for $i=1$, 2. Hence,

$$
\begin{aligned}
v \circ u(G) & =\left[v_{1}, v_{2}\right] \circ u(G) \\
& =\left[v_{1} \circ u, v_{2} \circ u\right](G) \\
& =\left[v_{1} \circ u(G), v_{2} \circ u(G)\right] .
\end{aligned}
$$

Thus $v \circ u(G)=v(u(G))$.

(ii) By Definition 2.4,

$v \circ u(G)=\left\langle v\left(u\left(g_{1}, \ldots, g_{t}\right), \ldots, u\left(g_{(s-1) t+1}, \ldots, g_{s t}\right)\right) ; g_{j} \in G, i\langle j\langle s t\rangle\right.$.

Put $b_{i}=u\left(g_{(i-1) t+1}, \ldots, g_{(i-1) t+k} a, \ldots, g_{i t}\right)\left[u\left(g_{(i-1) t+1}, \ldots, g_{i t}\right)\right]^{-1}$, where 1 $<i<s, 1<k<t$ and $a \in G$. Suppose $a \in(v \circ u)^{*}(G)$, then

$$
\begin{aligned}
v\left(u\left(g_{1}, \ldots, g_{t}\right), \ldots, b_{i} u\left(g_{(i-1) t+1}, \ldots, g_{i t}\right), \ldots, u\left(g_{(s-1) t+1}, \ldots, g_{s t}\right)\right) \\
=v\left(u\left(g_{1}, \ldots, g_{t}\right), \ldots, u\left(g_{(s-1) t+1}, \ldots, g_{s t}\right)\right) .
\end{aligned}
$$

As $b_{i} \in u(G)$, it implies that $b_{i} \in v^{*}(u(G))$ and hence $a \in H$. Conversely, if $a \in H$ then

$$
u\left(g_{1}, \ldots, g_{i} a, \ldots, g_{t}\right) \equiv u\left(g_{1}, \ldots, g_{t}\right) \bmod v^{*}(u(G)) .
$$

Thus $a \in(v \circ u)^{*}(G)$. 


\section{3. $p^{n}$-Schur-Baer property}

A polynilpotent word is defined as a composite of some nilpotent words as follows:

$$
\gamma_{c_{1}+1, \ldots, c_{t}+1}=\gamma_{c_{1}+1} \circ \gamma_{c_{2}+1} \circ \ldots \circ \gamma_{c_{1}+1}
$$

where $\gamma_{c_{i}+1}, 1 \leqslant i \leqslant l$, is a nilpotent word in distinct variables. The word $\gamma_{c_{1}+1}, \ldots, c_{l}+1$ is defined as the variety of polynilpotent groups of class row $\left(c_{1}, \ldots, c_{l}\right)$, which is denoted by $\mathbf{N}_{c_{1}, \ldots, c_{i}}$.

In this section we will prove the following main results.

THeOReM 3.1. Let $\mathbf{V}=\mathbf{N}_{r-1}$ be the variety of nilpotent groups of class at most $r-1$ and let $p$ be any prime number. Then $\mathrm{V}$ has the $p^{n}-S . B$. property.

THeOREM 3.2. Let $v=v\left(x_{1}, \ldots, x_{r}\right)$ and $u=u\left(x_{1}, \ldots, x_{s}\right)$ be two o.c. words defining the varieties $\mathrm{V}$ and $\mathrm{U}$, respectively. Let $w=v \circ u$, the composite of $v$ and $u$, define the variety $\mathbf{W}$. If the varieties $\mathbf{V}$ and $\mathbf{U}$ have the $p^{n}-S . B$. property, for every prime $p$, then so has the variety $\mathbf{W}$.

The above theorem can be easily extended to any number of o.c. words.

Now the following theorem is an immediate consequence of Theorems 3.1 and 3.2.

THEOREM 3.3. Let $\mathbf{V}=\mathbf{N}_{c_{1}, \ldots, c_{1}}$ be the variety of all polynilpotent groups of class row $\left(c_{1}, \ldots, c_{l}\right)$. Then $\mathrm{V}$ has the $p^{n}$-S.B. property, for every prime $p$.

In order to facilitate the proofs of the theorems, we shall prove some lemmas first. We shall also concentrate on varieties of groups defined by a single word in finitely many variables.

LeMmA 3.4. Let $v\left(x_{1}, \ldots, x_{r}\right)$ and $u\left(x_{r+1}, \ldots, x_{r+s}\right)$ be any two o.c. words in distinct variables defining the varieties $\mathrm{V}$ and $\mathrm{U}$, respectively. Let $\mathrm{W}$ be the variety defined by $w=[v, u]$. Then, for every group $G$ with a normal subgroup $N$

$$
\left[N W^{*} G\right]=\left[\left[N V^{*} G\right], U(G)\right]\left[V(G),\left[N U^{*} G\right]\right] \text {. }
$$

Proof. Clearly, $\left[N W^{*} G\right]$ is generated by the following set

$$
\begin{gathered}
S=\left\{\left[v\left(g_{1}, \ldots, g_{i-1}, b, g_{i+1}, \ldots, g_{r}\right), u\left(g_{r+1}, \ldots, g_{r+s}\right)\right] ;\right. \\
\left.g_{1}, \ldots, g_{r+s} \in G, 1 \leqslant i<r, b \in N\right\} \\
\cup\left\{\left[v\left(g_{1}, \ldots, g_{r}\right), u\left(g_{r+1}, \ldots, g_{r+j-1}, b, g_{r+j+1}, \ldots, g_{r+s}\right)\right] ;\right. \\
\left.g_{1}, \ldots, g_{r+s} \in G, 1<j<s, b \in N\right\},
\end{gathered}
$$


It is known that $\left[N V^{*} G\right]$ is generated by

$$
\left\{v\left(g_{1}, \ldots, g_{i-1}, b, g_{i+1}, \ldots, g_{r}\right) ; g_{i} \in G, 1<i<r, b \in N\right\},
$$

hence the first set in $S$ generates [[NV*G],U(G)]. Similarly, the second set in $S$ generates $\left[V(G),\left[N U^{*} G\right]\right]$.

The other containment is clear and hence the equality holds.

LEMMA 3.5. Let $F$ be a free group of countable rank and $w$ be an o.c. word of weight $c$ defining the variety $\mathrm{W}$. Then, for any prime number $p$

$$
\left[\left(F^{p} F^{\prime}\right) W^{*} F\right]=W(F)^{p}\left[F^{\prime} W^{*} F\right] .
$$

Proof. Suppose $w=[u, v]$, where $u$ and $v$ are o.c. words defining the varieties $\mathbf{U}$ and $\mathbf{V}$ respectively.

We prove by induction on the weight of $w$. For $c=2$, the result is obvious. Assume the assertion is true for all the o.c. words of weight less than $c$. Using Lemma 3.4,

$$
\left[\left(F^{p} F^{\prime}\right) W^{*} F\right]=\left[\left[\left(F^{p} F^{\prime}\right) U^{*} F\right], V(F)\right]\left[U(F),\left[\left(F^{p} F^{\prime}\right) V^{*} F\right]\right] .
$$

By induction hypothesis,

$$
\left[\left(F^{p} F^{\prime}\right) U^{*} F\right]=U(F)^{p}\left[F^{\prime} U^{*} F\right]
$$

and

$$
\left[\left(F^{p} F^{\prime}\right) V^{*} F\right]=V(F)^{p}\left[F^{\prime} V^{*} F\right] .
$$

Now, the result follows by using the commutator calculations, Lemma 3.4, and the fact that $\left[F^{\prime} W^{*} F\right]$ is normal in $F$.

Let $p$ be a prime number and let $Z_{p}$ denote the cyclic group of order $p$, and $Z_{p}^{n}$ be the direct product of $n$ copies of $Z_{p}$. Then the following proposition, which may be interesting in its own right, is true.

Proposition 3.6. Let $\mathrm{W}$ be a variety defined by an o.c. word $w$. Then the Baer-invariant of $Z_{p}^{n}$ is an elementary abelian $p$-group, for any prime number $p$ and any positive integer $n$.

Proof. Let $1 \rightarrow F^{p} F^{\prime} \rightarrow F \rightarrow Z_{p}^{n} \rightarrow 1$ be the free presentation for $Z_{p}^{n}$, where $F$ is the free group of rank $n$. By Lemma 3.5, and using the fact that $W(F)$ is contained in $F^{\prime}$

$$
\mathrm{W} M\left(Z_{p}^{n}\right)=\frac{W(F)}{\left[\left(F^{p} F^{\prime}\right) W^{*} F\right]}=\frac{W(F)}{W(F)^{p}\left[F^{\prime} W^{*} F\right]} .
$$

Hence the result holds. 
LEMMA 3.7. Let $\mathrm{V}$ be $a$ variety of groups and $G$ be $a$ finite group. Let $H=G / V^{*}(G)$ be the marginal factor-group of $G$. Then

$$
|V(G)|<|\mathbf{V} M(H)||V(H)| \text {. }
$$

Proof. Let

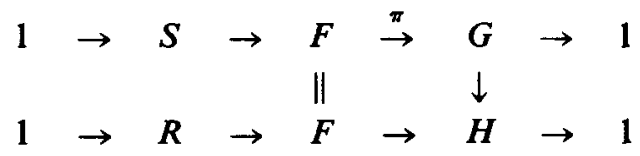

be free presentations for the groups $G$ and $H$ respectively, where $F$ is a free group. Clearly, the same free group $F$ can be chosen for both presentations, which makes the diagram commute and also $S$ will be contained in $R$. The epimorphism $\pi$ induces maps from $V(F)$ onto $V(G)$ and $R$ onto $V^{*}(G)$. Hence, using the fact that the image of $R$ in $G$ is marginal, $\left[R V^{*} F\right]$ is mapped onto the identity element of $G$.

We have $F / S \simeq G, F / R \simeq G / V^{*}(G)$ and $R / S \simeq V^{*}(G)$. Then by Lemma 2.3(i)

$$
\begin{gathered}
V(G) \simeq V\left(\frac{F}{S}\right)=\frac{V(F) S}{S}, \\
V^{*}(G) \cap V(G) \simeq \frac{R}{S} \cap \frac{V(F) S}{S}=\frac{(R \cap V(F)) S}{S} .
\end{gathered}
$$

Thus $V^{*}(G) \cap V(G)$ is a homomorphic image of $R \cap V(F)$ and hence the Baer-invariant of $H, \operatorname{VM}(H)$, will be mapped onto $V^{*}(G) \cap V(G)$. Therefore

$$
\left|V^{*}(G) \cap V(G)\right|<|V M(H)| .
$$

By Lemma 2.3(i) and the isomorphism Theorem,

$$
V(H)=V\left(\frac{G}{V^{*}(G)}\right) \simeq \frac{V(G)}{V^{*}(G) \cap V(G)} .
$$

Hence $|V(G)|=\left|V^{*}(G) \cap V(H)\right||V(H)|$, or $|V(G)|<|V M(H)||V(H)|$.

The following results are purely technical.

LEMMA 3.8: Let $\mathrm{V}$ be a variety of groups containing the variety of abelian groups. Let $F$ be the free group with the free set of generators $\left\{x_{1}, x_{2}, \ldots, x_{n-1}, x\right\}$. Then

$$
V(F) \cap \overline{\langle x\rangle}=V(F) \cap[\langle x\rangle, F]=\left[\overline{\langle x\rangle} V^{*} F\right],
$$

Proof. Clearly $\overline{\langle x\rangle}=\langle x\rangle^{F}=\langle x\rangle[\langle x\rangle, F]$ and hence

$$
V(F) \cap \overline{\langle x\rangle}=V(F) \cap[\langle x\rangle, F],
$$

since by the assumption $V(F)$ is contained in $F$. The inclusion $\left[\langle x\rangle V^{*} F\right] \subset$ $V(F) \cap\langle\bar{x}\rangle$ follows by Lemma $2.4($ iii). 
Conversely, let $g$ be an element of $V(F)$ then $g$ can be written as $g=$ $v\left(x_{1}, \ldots, x_{n-1}, x\right)$, where $v$ is a law in $V$. Now if $g$ is also in $[\langle x\rangle, F]$, then we must have $v\left(x_{1}, \ldots, x_{n-1}, 1\right)=1$. Thus

$$
g=v\left(x_{1}, \ldots, x_{n-1}, 1 x\right)\left(v\left(x_{1}, \ldots, x_{n-1}, 1\right)\right)^{-1},
$$

which, by definition, belongs to $\left[\overline{\langle x\rangle} V^{*} F\right]$ and hence the inclusion follows the other way round.

LEMMA 3.9. Let $\mathbf{V}=\mathbf{N}_{r-1}$ be the variety of nilpotent groups of class at most $r-1(r>1)$ and $G$ be a group. Let $y$ be a fixed element of $G$ and $p$ be a prime number. If $y^{p}$ and $[y, g]$ are in $V^{*}(G)$, for all $g$ in $G$, then

$$
\left[g_{1}, \ldots, g_{i-1}, y, g_{i+1}, \ldots, g_{r+1}\right]=1
$$

and

$$
\left[g_{1}, \ldots, g_{i-1}, y, g_{i+1}, \ldots, g_{r}\right]^{p}=1,
$$

for all $g_{1}, \ldots, g_{r+1}$ in $G$ and all $i$.

Proof. By the assumption and the definition of marginality in the variety of nilpotent groups,

$$
\left[g_{1}, \ldots, g_{i-1},[y, g], g_{i+1}, \ldots, g_{r}\right]=1
$$

and

$$
\left[g_{1}, \ldots, g_{i-1}, y^{p}, g_{i+1}, \ldots, g_{r}\right]=1,
$$

for all $i$ and all $g, g_{j}$ in $G, j=1, \ldots, r$. The proof goes by induction on $i$. For $i=1$, then $\left[y, g, g_{2}, \ldots, g_{r}\right]=1$ and $\left[y^{p}, g_{2}, \ldots, g_{r}\right]=1$, for all $g, g_{j}$ in $G$. By Lemma 2.1(i), and the first identity of the lemma, $\left[y, g_{2}, \ldots, g_{r}\right]^{p}=1$.

By the Witt identity and routine commutator calculations,

$$
\begin{aligned}
{\left[g_{1}, \ldots, g_{i-1}, y,\right.} & \left.g_{i+1}, \ldots, g_{r+1}\right] \\
= & {\left[g_{1}^{\prime}, \ldots, g_{i-2}^{\prime},\left[y, g_{i-1}^{\prime}\right], g_{i+1}^{\prime}, \ldots, g_{r+1}^{\prime}\right]^{h_{1}} } \\
& \cdot\left[g_{1}^{\prime \prime}, \ldots, g_{i-2}^{\prime \prime}, y, g_{i-1}^{\prime \prime}, g_{i+1}^{\prime \prime}, \ldots, g_{r+1}^{\prime \prime}\right]^{h_{2}},
\end{aligned}
$$

where $g_{j}^{\prime}$ and $g_{j}^{\prime \prime}$ are some conjugates of $g_{j}$, for $j=1, \ldots, i-1, i+1, \ldots, r+$ 1 , and $h_{1}, h_{2}$ are some elements of $G$. But the first factor is trivial, since $\left[y, g_{i-1}^{\prime}\right]$ is marginal, and the second one is also trivial by the induction hypothesis.

Now, the second relation is an immediate consequence of the first one. Using Lemma 2.1(ii),

$$
\left[g_{1}, \ldots, g_{i-1}, y^{p}, g_{i+1}, \ldots, g_{r}\right]=\left[g_{1}, \ldots, g_{i-1}, y, g_{i+1}, \ldots, g_{r}\right]^{p} \cdot k,
$$

where $k$ is the product of commutators of weights $>r+1$ with at least one $y$ as an entry in each one. These are all trivial by the first identity. 
Proposinon 3.10. Let $\mathbf{V}=\mathbf{N}_{r-1}$ be the variety of nilpotent groups of class at most $r-1$. Let $F$ be a free group of countable rank and $\mathrm{W}$ be the variety defined by $W(F)=\left[\left(F^{p} F^{\prime}\right) V^{*} F\right], p$ is any prime number. If $y$ is an element of a group $G$ such that $[y, g]$ and $y$ are in $V^{*}(G)$, for all $g \in G$, then $y \in W^{*}(G)$.

Proof. By Lemma 3.5, and using the assumption that $\mathrm{V}$ is the variety of nilpotent groups,

$$
W(F)=(V(F))^{p}\left[F^{\prime} V^{*} F\right]=\left(\gamma_{r}(F)\right)^{p} \gamma_{r+1}(F) .
$$

Therefore, $W(G)$ is generated by the following set of elements,

$$
\left\{\left[g_{1}, \ldots, g_{r}\right]^{p},\left[g_{1}, \ldots, g_{r+1}\right] ; g_{i} \in G, 1<i<r+1\right\} .
$$

By the assumption $[y, g]$ and $y^{p}$ are in $V^{*}(G)$, hence Lemma 3.9 implies that

$$
\left[g_{1}, \ldots, g_{i-1}, y, g_{i+1}, \ldots, g_{r+1}\right]=1
$$

and

$$
\left[g_{1}, \ldots, g_{i-1}, y, g_{i+1}, \ldots, g_{r}\right]^{p}=1,
$$

for all $g_{1}, \ldots, \mathrm{g}_{r+1}$ in $G$ and all $i$.

Now, to show that $y$ is marginal in $G$ with respect to the variety $\mathbf{W}$, it remains to prove the following:

$$
\left[g_{1}, \ldots, g_{i-1}, g_{i} y, g_{i+1}, \ldots, g_{r}\right]^{p}=\left[g_{1}, \ldots, g_{i}, \ldots, g_{r}\right]^{p},
$$

for all $g_{1}, \ldots, g_{r}$ in $G$ and all $i$. This is proved by using the above identities and repeated application of commutator identities. Thus $y$ is in $W^{*}(G)$.

Let $\mathbf{V}$ be a variety of groups containing the variety of abelian groups $\mathbf{A}$. Let

$$
Z_{p}^{n}=Z_{p}\left(a_{1}\right) \times \cdots \times Z_{p}\left(a_{n}\right),
$$

where $Z_{p}\left(a_{i}\right)$ is a cyclic group of order $p$ and generated by the elements $a_{i}$, for each $i$, and $Z_{p}^{n-1}=\left\langle a_{1}, \ldots, a_{n-1}\right\rangle \subset Z_{p}^{n}$. Let

$$
\begin{aligned}
& 1 \rightarrow S \rightarrow F \stackrel{\pi}{\rightarrow} \underset{\substack{\downarrow_{p}^{n} \\
\downarrow \alpha}}{Z^{n}} \rightarrow 1 \\
& 1 \rightarrow \hat{S} \rightarrow \hat{F} \stackrel{\hat{\pi}}{\rightarrow} Z_{p}^{n-1} \rightarrow 1
\end{aligned}
$$

be the free presentations for $Z_{p}^{n}$ and $Z_{p}^{n-1}$ respectively, where $F$ is free on $\left\{x_{1}, \ldots, x_{n}\right\}$ and $\hat{F}$ on $\left\{x_{1}, \ldots, x_{n-1}\right\}$ and $S=F^{\prime} F^{p}, \hat{S}=\hat{F}^{\prime} \hat{F}^{p}, x_{i} \pi=a_{i}, x_{i} \hat{\pi}=$ $a_{i}$. Now, let $\alpha$ be the homomorphism from $Z_{p}^{n}$ onto $Z_{p}^{n-1}$ given by $a_{i} \alpha=a_{i}$, for all $i \neq n$, and $a_{n} \alpha=1$. Then $\alpha$ is induced by the epimorphism $\beta: F \rightarrow \hat{F}$ such that $x_{i} \beta=x_{i}$, for all $i \neq n$, and $x_{n} \beta=1$. This gives rise to a homomorphism $\alpha^{\prime}$ 
from the Baer-invariant of $Z_{p}^{n}$ onto the Baer-invariant of $Z_{p}^{n-1}$, that is,

$$
\alpha^{\prime}: \frac{V(F)}{\left[S V^{*} F\right]} \rightarrow \frac{V(\hat{F})}{\left[\hat{S} V^{*} \hat{F}\right]},
$$

which is independent of $\beta$ (see Moghaddam (1975) or Leedham-Green and McKay (1976)). Clearly

$$
\operatorname{ker} \alpha^{\prime}=\frac{\left(V(F) \cap \overline{\left\langle x_{n}\right\rangle}\right)\left[S V^{*} F\right]}{\left[S V^{*} F\right]} .
$$

Now the following technical lemma shortens the proof of Theorem 3.1.

LEMMA 3.11. Let $\mathrm{W}$ be the variety of groups defined by $W(F)=\left[\left(F^{\prime} F^{p}\right) V^{*} F\right]$. With the above assumptions and notations if $K=\operatorname{ker} \alpha^{\prime}$, then

$$
K \simeq \frac{\left[\overline{\left\langle x_{n}\right\rangle} V^{*} F\right]}{\left[\overline{\left\langle x_{n}\right\rangle} W^{*} F\right]}
$$

Proof. By the above discussion, and using the fact that $W(F)$ is contained in $V(F)$

$$
\begin{aligned}
K & =\frac{\left(V(F) \cap \overline{\left\langle x_{n}\right\rangle}\right)\left[\left(F^{\prime} F^{p}\right) V^{*} F\right]}{\left[\left(F^{\prime} F^{p}\right) V^{*} F\right]} \\
& \simeq \frac{V(F) \cap \overline{\left\langle x_{n}\right\rangle}}{W(F) \cap \overline{\left\langle x_{n}\right\rangle}} .
\end{aligned}
$$

Hence by Lemma 3.8,

$$
K \simeq \frac{\left[\overline{\left\langle x_{n}\right\rangle} V^{*} F\right]}{\left[\overline{\left\langle x_{n}\right\rangle} W^{*} F\right]}
$$

Now we are able to give the proofs of the theorems.

Proof of Theorem 3.1. Since a good deal of the proof goes through for a bigger variety, we shall first assume that $\mathbf{V}$ is the variety defined by the set of o.c. words $V$.

Now for any group $G$ with $G / V^{*}(G)=H$, say, of order $p^{n}$, we need to show that

$$
|V(G)|<\left|\mathbf{V} M\left(Z_{p}^{n}\right)\right| .
$$

We proceed by induction on $n$. For $n=0$, then $G=V^{*}(G)$ and hence by Lemma 2.2(i)

$$
V(G)=\langle 1\rangle .
$$


If $n=1$, then $H$ is a cyclic group of order $p$. Clearly, when the variety is defined by commutator words only, then the verbal subgroup as well as the Baerinvariant of any cyclic group is trivial. So, Lemma 3.7 implies that $V(G)=\langle 1\rangle$.

Now, assume that the result holds for all groups with marginal factor-group of order $p^{m}$, for all $m<n$. Choose $y$ in $G \backslash V^{*}(G)$ such that $\bar{y}=y V^{*}(G)$ is central and of order $p$ in $H$, that is, $[y, g]$ and $y^{p}$ are in $V^{*}(G)$, for all $g$ in $G$. Let

$$
N=\left\langle v\left(g_{1}, \ldots, g_{i-1}, y, g_{i+1}, \ldots, g_{r}\right) ; g_{j} \in G \backslash V^{*}(G), v \in V, i\langle i\langle r\rangle \text {. }\right.
$$

By Theorem 2.3 of Turner-Smith (1964), $N^{G}=\left[\overline{\langle y\rangle} V^{*} G\right]$, where $\overline{\langle y\rangle}$ is the normal closure of $\langle y\rangle$ in $G$. Clearly, by using the fact that $[y, g]$ is in $V^{*}(G)$, for all $g$ in $G$, it implies that $N$ is normal in $G$ and is contained in $V^{*}(G)$. Hence $N=\left[\overline{\langle y\rangle} V^{*} G\right]$.

Set $G_{1}=G / N$, then $V^{*}\left(G_{1}\right) \supsetneqq V^{*}(G) / N$. For, by definition of $N$

$$
\begin{aligned}
v\left(g_{1} N, \ldots, g_{i-1} N, y N,\right. & \left.g_{i+1} N, \ldots, g_{r} N\right) \\
& =v\left(g_{1}, \ldots, g_{i-1}, y, g_{i+1}, \ldots, g_{r}\right) N=N .
\end{aligned}
$$

Hence $y N \in V^{*}\left(G_{1}\right)$, where as $y \notin V^{*}(G)$. Thus

$$
\left|\frac{G_{1}}{V^{*}\left(G_{1}\right)}\right|<\left|\frac{G / N}{V^{*}(G) / N}\right|=p^{n}
$$

so $\left|G_{1} / V^{*}\left(G_{1}\right)\right| \leqslant p^{m}$, for some $m<n$. Now $n-1$ is the maximum value of $m$ and by induction hypothesis,

$$
\left|V\left(G_{1}\right)\right|<\left|\mathbf{V} M\left(Z_{p}^{n-1}\right)\right|
$$

By Lemma 2.2(i) and the fact that $N$ is contained in $V(G)$ we have,

$$
V\left(G_{1}\right)=V\left(\frac{G}{N}\right)=\frac{V(G)}{N} .
$$

Therefore $|V(G)|=\left|V\left(G_{1}\right)\right||N|$. It is enough to show that

$$
|N|<\frac{\left|\mathbf{V} M\left(Z_{p}^{n}\right)\right|}{\left|\mathbf{V} M\left(Z_{p}^{n-1}\right)\right|}=|K| \text {, say. }
$$

By Lemma 3.11, we have

$$
|K|=\left|\frac{\left[\overline{\left\langle x_{n}\right\rangle} V^{*} F\right]}{\left[\overline{\left\langle x_{n}\right\rangle} W^{*} F\right]}\right|,
$$

where $F$ is the free group on $\left\{x_{1}, \ldots, x_{n}\right\}$ and the variety $\mathbf{W}$ is as defined in Lemma 3.11 .

Let $\left\{\bar{g}_{1}, \ldots, \bar{g}_{n-1}, \bar{y}\right\}$ be a generating set for the $p$-group $H$, where $\bar{y}$ is as before and $\bar{g}_{i}=g_{i} V^{*}(G)$, for some $g_{i}$ in $G(1<i<n-1)$. There exist a homomorphism $\theta$ of $F$ into $G$ such that $x_{i} \theta=g_{i}$, for all $i \neq n$, and $x_{n} \theta=y$. Since the group $G$ is generated by $\left\{g_{1}, \ldots, g_{n-1}, y\right\} \cup V^{*}(G), \theta$ induces a 
mapping from $\left[\overline{\left\langle x_{n}\right\rangle} V^{*} F\right]$ onto $\left[\overline{\langle y\rangle} V^{*} G\right]$. Similarly, $\theta$ induces a mapping from $\left[\overline{\left\langle x_{n}\right\rangle} W^{*} F\right]$ onto $\left[\overline{\langle y\rangle} W^{*} G\right]$.

From now on we assume that $\mathbf{V}$ is the variety of nilpotent groups of class $r-1$. Then $\theta$ induces a mapping from $K$ onto $\left[\overline{\langle y\rangle} V^{*} G\right]$, for the proposition 3.10 implies that $y$ is marginal in $G$ with respect to the variety $\mathbf{W}$, and hence $\left[\overline{\langle y\rangle} W^{*} G\right]=1$. Thus $|N|<|K|$ and the proof is completed.

Proof of Theorem 3.2. Let $G$ be a group with $G / W^{*}(G)$ of order $p^{n}$. By Lemma 2.5(ii)

$$
\frac{W^{*}(G)}{V^{*}(U(G))}=U^{*}\left(\frac{G}{V^{*}(U(G))}\right)
$$

Let $K=G / V^{*}(U(G))$. Then clearly $K / U^{*}(K) \simeq G / W^{*}(G)$. Hence $\left|K / U^{*}(K)\right|=p^{n}$ and by assumption for the variety $\mathrm{U}$,

$$
|U(K)|<\left|\mathbf{U} M\left(Z_{p}^{n}\right)\right|=p^{m} \text {, say. }
$$

Using Proposition 3.6 we note that $\mathrm{U} M\left(Z_{p}^{n}\right)$ is an elementary abelian $p$-group, and Lemma 2.3(i) implies that

$$
U(K)=\frac{U(G)}{V^{*}(U(G))}
$$

Now, by the assumption for the variety $\mathbf{V}$ and the fact that the marginal factor-group is $U(K)$, we have

$$
|V(U(G))|<\left|\mathbf{V} M\left(\mathbf{U} M\left(Z_{p}^{n}\right)\right)\right|
$$

But by Lemma 2.5(i), $W(G)=V(U(G))$. Hence it remains to show that

$$
\left|\mathbf{V} M\left(\mathbf{U} M\left(Z_{p}^{n}\right)\right)\right|<\left|\mathbf{W} M\left(Z_{p}^{n}\right)\right| \text {. }
$$

Let

$$
1 \rightarrow S=F^{\prime} F^{p} \rightarrow F \rightarrow Z_{p}^{n} \rightarrow 1
$$

be the free presentation for $Z_{p}^{n}$, where $F$ is the free group on $\left\{x_{1}, \ldots, x_{n}\right\}$. Using the fact that the varieties $\mathbf{W}$ and $\mathbf{U}$ contain the variety of abelian groups, then the Baer-invariants of $Z_{p}^{n}$ with respect to the varieties $\mathbf{W}$ and $\mathbf{U}$ are

$$
\begin{gathered}
\mathbf{W} M\left(Z_{p}^{n}\right)=\frac{W(F)}{\left[S W^{*} F\right]}=\frac{V(U(F))}{\left[S W^{*} F\right]}, \\
\mathbf{U} M\left(Z_{p}^{n}\right)=\frac{U(F)}{\left[S U^{*} F\right]},
\end{gathered}
$$

respectively.

Now

$$
1 \rightarrow S_{1} \rightarrow U(F) \rightarrow \mathbf{U} M\left(Z_{p}^{n}\right) \rightarrow 1
$$


is a free presentation for $\mathrm{UM}\left(Z_{p}^{n}\right)$, where $S_{1}=\left[S U^{*} F\right]$. Hence the Baerinvariant of $U M\left(Z_{p}^{n}\right)$ with respect to the variety $V$ is

$$
\operatorname{VM}\left(\mathbf{U} M\left(Z_{p}^{n}\right)\right)=\frac{S_{1} \cap V(U(F))}{\left[S_{1} V^{*} U(F)\right]}
$$

Knowing that $u$ is an o.c. word, then $S_{1}$ is generated by the following set, say $T_{1}$,

$$
T_{1}=\left\{u\left(f_{1}, \ldots, f_{i-1}, t, f_{i+1}, \ldots, f_{s}\right) ; f_{j} \in F, 1<i, j<s, t \in S\right\} .
$$

By Definition 2.4 and bearing in mind that the composite of two o.c. words is an o.c. word, $\left[S W^{*} F\right]$ is generated by

$$
\begin{aligned}
\left\{v \left(u\left(f_{1}, \ldots, f_{s}\right), \ldots,\right.\right. & u\left(f_{(i-1) s+1}, \ldots, f_{j-1}, t, f_{j+1}, \ldots, f_{i s}\right), \ldots, \\
& \left.\left.u\left(f_{(r-1) s+1}, \ldots, f_{r s}\right)\right) ; f_{1}, \ldots, f_{r s} \in F, t \in S \text { and all } i, j\right\} .
\end{aligned}
$$

Also $\left[S_{1} V^{*} U(F)\right]$ is generated by

$$
\left\{v\left(u_{1}, \ldots, u_{i-1}, g, u_{i+1}, \ldots, u_{r}\right) ; u_{1}, \ldots, u_{r} \in U(F), g \in T_{1}, 1<i<r\right\},
$$

which is the set of generators of $\left[S W^{*} F\right]$.

Now by comparing (1) and (2), we get

$$
\mathbf{V} M\left(\mathbf{U} M\left(Z_{p}^{n}\right)\right) \subset \mathbf{W} M\left(Z_{p}^{n}\right)
$$

which completes the proof.

The next corollary generalizes the result of M. Jones (1972) for the Schurmultiplicator of a finite $p$-group to the Baer-invariant.

COROLlaRY 3.12. Let $\mathbf{V}=\mathbf{N}_{c_{1}, \ldots, c_{l}}$ be the variety of polynilpotent groups and $G$ be a p-group of order $p^{n}$, where $p$ is any prime number. Then

$$
|V(G)||\mathbf{V} M(G)|<\left|\mathbf{V} M\left(Z_{p}^{n}\right)\right| \text {. }
$$

Proof. Let $G=F / R$ be a free presentation for the group $G$, where $F$ is the free group of rank $n$. Let $H=F /\left[R V^{*} F\right]$, then Lemma 2.3(ii) implies that

$$
\frac{R}{\left[R V^{*} F\right]} \subseteq V^{*}\left(\frac{F}{\left[R V^{*} F\right]}\right) \text {. }
$$

Hence

$$
\left|\frac{H}{V^{*}(H)}\right|<|G|=p^{n} \text {. }
$$

Now applying Theorem 3.3., $|V(H)|<\left|V M\left(Z_{p}^{n}\right)\right|$. But

$$
|V(H)|=\left|\frac{V(F)}{\left[R V^{*} F\right]}\right|=\left|\frac{V(F)}{R \cap V(F)}\right|\left|\frac{R \cap V(F)}{\left[R V^{*} F\right]}\right|,
$$


and by Lemma 2.3(i) and the Isomorphism Theorem,

$$
V(G)=V\left(\frac{F}{R}\right) \simeq \frac{V(F)}{R \cap V(F)} .
$$

Thus the result holds.

\section{Attaining the bound}

In this final section, we construct an example, Theorem 4.3, to show that the bound, which has been obtained in Theorem 3.3, is attained.

In order to construct the example, we prove the following lemmas.

LEMMA 4.1. Let $\mathbf{V}=\mathbf{N}_{c_{1}, \ldots, c_{1}}$ be a variety of polynilpotent groups defined by the polynilpotent word $\gamma_{c_{1}+1, \ldots, c_{t}+1}$, and let $p$ be any prime number. Then the Baer-invariant of $Z_{p}^{n}$ is a non-trivial elementary abelian p-group for $n>1$, except in the case when $n=2$ and there exists $i$ with $l>i>1$ such that $c_{l}=c_{l-1}$ $=\cdots=c_{i+1}=2$ and $c_{i}=1$.

Proof. Let

$$
1 \rightarrow F^{p} F^{\prime} \rightarrow F \rightarrow Z_{p}^{n} \rightarrow 1
$$

be the free presentation for $Z_{p}^{n}$, where $F$ is the free group of rank $n$.

By Proposition 3.6, the Baer-invariant of $Z_{p}^{n}$ with respect to a variety defined by o.c. words is

$$
\mathbf{V} M\left(Z_{p}^{n}\right)=\frac{V(F)}{(V(F))^{p}\left[F^{\prime} V^{*} F\right]}
$$

which is an elementary abelian $p$-group.

To prove the non-triviality, we start by induction on $l$. If $l=1$, then commutator manipulations imply

$$
\mathbf{N}_{c_{1}} M\left(Z_{p}^{n}\right)=\frac{F_{c_{1}+1}}{\left[F^{p} F^{\prime}, F_{c_{1}}\right]}=\frac{F_{c_{1}+1}}{F_{c_{1}+1}^{p} F_{c_{1}+2}},
$$

which is of order $p^{f\left(c_{1}, n\right)}$, where $f\left(c_{1}, n\right)$ is the number of basic commutators of weight $c_{1}+1$ on $n$ generators. Note that $f\left(c_{1}, n\right)>3$ except that $f(1,2)=1$ and $f(2,2)=2$.

Suppose $n>2$ or $n=2$ and $c_{l}>2$, then assume that the Baer-invariant of $Z_{p}^{n}$ is non-trivial with respect to all the polynilpotent varieties of class row $\left(c_{2}, \ldots, c_{l}\right)$ and that there are at least three basic commutators among the set of generators of the same shape as $\gamma_{c_{2}+1, \ldots, c_{t}+1}$. 
Now $\gamma_{c_{1}+1}\left(\gamma_{c_{2}+1, \ldots, c_{1}+1}(F)\right)$ is the verbal subgroup of $F$, which occurs in the Baer-invariant of $Z_{p}^{n}$ with respect to $\mathbf{N}_{c_{1}, \ldots, c_{i}}$. By induction hypothesis, we can arrange and build up at least three basic commutators in $n$ generators of the required form.

In the case $n=2$ and $c_{l}=2$, if $c_{i+1}=\cdots=c_{l-1}=c_{l}=2$ then we start by having two basic commutators of weight three in two generators. Thus we are able to build two basic commutators in two generators of the same shape as the polynilpotent word at each stage down to $i+1$. Now, if $c_{j}=1$ then we get only one basic commutator of the same shape as $\gamma_{2,3, \ldots, 3}$ (where 3 occurs $l-i-1$ times) and at this stage the only case allowed is $i=1$. But, if $c_{i}>2$ then we get more than two basic commutators of the required form and we can proceed as before.

The following lemma deals with the Baer-invariant of $Z_{p}^{2}$, the case that has been excluded in Lemma 4.1.

LEMMA 4.2. The Baer-invariant of $Z_{p}^{2}$ is a cyclic group with respect to the variety of polynilpotent groups of class row $(1,2, \ldots, 2)$ (where 2 occurs $l-1$ times), and it is trivial when the given class row is

$$
\left(c_{1}-1, \ldots, c_{j}-1,1,2, \ldots, 2\right), \quad j>1,
$$

where 2 occurs $l-1$ times.

Proof. Let

$$
1 \rightarrow F^{p} F^{\prime} \rightarrow F \rightarrow Z_{p}^{n} \rightarrow 1
$$

be the free presentation for $Z_{p}^{n}$, where $F$ is the free group of rank $n$. Let $\mathbf{U}=\mathbf{N}_{c_{2}-1, \ldots, c_{1}-1}$ and $\mathbf{V}=\mathbf{N}_{c_{1}-1, \ldots, c_{1}-1}$ be two varieties of polynilpotent groups defined by the laws $\gamma_{c_{2}}, \ldots, c_{t}$ and $\gamma_{c_{1}}, \ldots, c_{i}$, respectively. Let $K_{1}$ and $K_{2}$ denote $\left(\gamma_{c_{2}, \ldots, c_{1}}(F)\right)^{p}\left[F^{\prime} U^{*} F\right]$ and $\left(\gamma_{c_{1}}\left(\gamma_{c_{2}, \ldots, c_{1}}(F)\right)\right)^{p}\left[F^{\prime} V^{*} F\right]$, respectively. Then by Proposition 3.6,

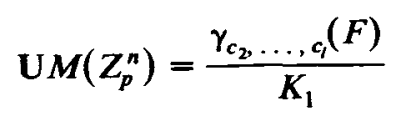

and

$$
\mathbf{V} M\left(Z_{p}^{n}\right)=\frac{\gamma_{c_{1}}\left(\gamma_{c_{2}, \ldots, c_{1}}(F)\right)}{K_{2}}
$$

Suppose $\mathbf{U} M\left(Z_{p}^{n}\right)$ is generated by the following set:

$$
\left\{y_{1} K_{1}, \ldots, y_{r} K_{1} ; y_{t} \in \gamma_{c_{2}}, \ldots, c_{t}(F), 1<t<r\right\} \text {. }
$$

Let

$$
S=\left\{\left[y_{i_{1}} K_{1}, \ldots, y_{i_{1}} K_{1}\right] ; 1<i_{j} \leqslant r, 1 \leqslant j<c_{1}\right\}
$$


Now, by using the routine commutator calculations one can easily see that all the commutators of weight $c_{1}$ in $S$, which involve at least one $K_{1}$ as an entry, are contained in $K_{2}$. Hence $\gamma_{c_{1}}, \ldots, c_{l}(F)$ is generated, modulo $K_{2}$, by

$$
\left\{\left[y_{i_{1}}, \ldots, y_{i_{1}}\right] ; 1<i_{j} \leqslant r, 1<j<c_{1}\right\} \text {. }
$$

From now on assume $n=2, c_{2}=3, \ldots, c_{l}=3$ and $c_{1}=2$. Then if $\mathbf{N}_{2, \ldots,{ }_{2}} M\left(Z_{p}^{2}\right)$ ( $n-1$ twos) is generated by the two basic commutators $y_{1}$ and $y_{2}$ with $y_{1}<y_{2}$, it is clear from the above discussion that $\left[y_{2}, y_{1}, y_{1}\right]$ and $\left[y_{2}, y_{1}, y_{2}\right]$ generate $\mathbf{N}_{2} \ldots, 2 M\left(Z_{p}^{2}\right)(m-1$ twos $), 2 \leqslant m \leqslant l$. These clearly are basic in the two generators and so are independent. Thus $N_{2, \ldots, 2} M\left(Z_{p}^{2}\right)(l-1$ twos) requires exactly two generators. Now, by applying the abelian law it gives that $\mathbf{N}_{1,2, \ldots, 2_{2}} M\left(Z_{p}^{2}\right)(l-1$ twos) is generated by the only basic commutator of the right shape and hence it is cyclic.

Clearly, when the given class row is $\left(c_{1}-1, \ldots, c_{j}-1,1,2, \ldots, 2\right), j>1$, where 2 occurs $l-1$ times, then the Baer-invariant is trivial and hence the proof is completed.

Now, we are in a position to construct the example.

THEOREM 4.3. Let $v\left(x_{1}, \ldots, x_{n}\right)$ be a polynilpotent word defining the variety of polynilpotent groups $\mathrm{V}$ with the given class row $\left(c_{1}-1, \ldots, c_{l}-1\right)$, say. Then for any prime $p$ and non-negative integer $n$ different from 1 , there exists a group $G$ such that $G / V^{*}(G)$ is of order $p^{n}$ and $|V(G)|=\left|\mathrm{V} M\left(Z_{p}^{n}\right)\right|$, except when $n=2$ and the class row of polynilpotency is $\left(c_{1}-1, \ldots, c_{j}-1,1,2, \ldots, 2\right), j>1$, where 2 occurs $l-j-1$ times.

Proof. Let

$$
1 \rightarrow S \rightarrow F \rightarrow Z_{p}^{n} \rightarrow 1
$$

be the free presentation for $Z_{p}^{n}$, where $F$ is the free group on $\left\{x_{1}, \ldots, x_{n}\right\}$ and $S=F^{p} F^{\prime}$. Then clearly,

$$
\mathbf{V} M\left(Z_{p}^{n}\right)=\frac{V(F)}{\left[S V^{*} F\right]} .
$$

Put $G=F /\left[S V^{*} F\right]$, then by Lemma 2.3(i)

$$
V(G)=\frac{V(F)}{\left[S V^{*} F\right]}
$$

and so $|V(G)|=\left|\mathrm{V} M\left(Z_{p}^{n}\right)\right|$. Now, we need to prove that $G / V^{*}(G)$ is of order $p^{n}$, and hence it is sufficient to show that

$$
\frac{S}{\left[S V^{*} F\right]}=V^{*}\left(\frac{F}{\left[S V^{*} F\right]}\right) \text {. }
$$


Lemma 2.3(ii) implies the inclusion

$$
\frac{S}{\left[S V^{*} F\right]} \subseteq V^{*}\left(\frac{F}{\left[S V^{*} F\right]}\right),
$$

for any variety $\mathbf{V}$.

Conversely, by Lemma 3.5

$$
\left[S V^{*} F\right]=\left[\left(F^{p} F^{\prime}\right) V^{*} F\right]=(V(F))^{p}\left[F^{\prime} V^{*} F\right] .
$$

It is enough to show that any element $w$ in $F \backslash F^{p} F^{\prime}$ is not marginal in $F /\left[S V^{*} F\right]$.

Now $F / F^{p} F^{\prime}$ is a vector space over a field of $p$ elements, so it has a basis $\left\{x_{1}, \ldots, x_{n}\right\}$, say. Let $w \in F / F^{p} F^{\prime}$, then $w$ has a unique representation as follows:

$$
w=x_{1}^{\alpha_{1}} \cdots x_{n}^{\alpha_{n}}, \quad \text { where } 0<\alpha_{i}<p .
$$

If the greatest common divisor of $\alpha_{1}, \ldots, \alpha_{n}$ is $\beta$, then put

$$
w_{1}=x_{1}^{\alpha_{1} / \beta} \ldots x_{n}^{\alpha_{n} / \beta} \text {. }
$$

Now we can choose a basis for $F / F^{p} F^{\prime}$ from the set of $\left\{w_{1}, x_{1}, \ldots, x_{n}\right\}$ by omitting some $x_{i}$, say $x_{1}$. Hence $\left\{w_{1}, x_{2}, \ldots, x_{n}\right\}$ is a basis for $F / F^{p} F^{\prime}$. Thus we can always choose a free generating set for the free group $F,\left\{y_{1}, \ldots, y_{n}\right\}$ say, so that $w$ is a power of $y_{t}$, say, that is $w=y_{t}^{k}(0<k<p, 1<t<n)$.

By Lemma 4.1, in the case that $\operatorname{VM}\left(Z_{p}^{n}\right)$ is non-trivial, we can always choose a basis commutator in $n$ generators of the same shape as polynilpotent law, $v\left(y_{i_{1}}, \ldots, y_{i_{i}}\right)$ say, $\left(y_{i_{j}} \in\left\{y_{1}, \ldots, y_{n}\right\}\right)$ which is not in $(V(F))^{p}\left[F^{\prime} V^{*} F\right]$. In this basic commutator, suppose $y_{i_{j}}=y_{t}$ and replace $y_{t}$ by $y_{t}^{k}$ then the commutator manipulations imply

$$
\begin{aligned}
v\left(y_{i_{1}}, \ldots, y_{j_{-1}}, y_{t}^{k}, y_{i_{j+1}}, \ldots, y_{i}\right) & \\
& \equiv v\left(y_{i_{1}}, \ldots, y_{i_{-1}}, y_{t}, y_{i_{j+1}}, \ldots, y_{i_{r}}\right)^{k} \neq 1 \bmod (V(F))^{p}\left[F^{\prime} V^{*} F\right],
\end{aligned}
$$

since $0<k<p$, as required.

If $n=2$ and the class row of polynilpotency is

$$
\left(c_{1}-1, \ldots, c_{j}-1,1,2, \ldots, 2\right) \text {, }
$$

where 2 occurs $l-j-1$ times, then by Lemma 4.2 the Baer-invariant of $Z_{p}^{2}$ is trivial. Hence $V(G)=\langle 1\rangle$ and by Lemma 2.2(i), $G=V^{*}(G)$. In fact it follows from Theorem 3.3 that there are no groups with marginal factor of order $p^{2}$ for these varieties.

Finally, we remark that Theorem 2.1 of Wiegold (1965) is a particular case of Theorem 3.3, for the variety of abelian groups. Also, in the same paper Wiegold 
(1965) constructed an example showing that the bound is attained. This result is also a consequence of Theorem 4.3 , for the variety of abelian groups.

The author conjectures that Theorem 3.3., and hence the other relevant results, hold for the varieties defined by o.c. words.

\section{References}

J. A. Green (1956), 'On the number of automorphisms of a finite group', Proc. Roy. Soc. London Ser. A 237, 574-581.

J. A. Hulse and J. C. Lennox (1976), 'Marginal series in groups', Proc. Roy. Soc. Edinburgh, 67 Sect. A, 139-154.

M. Hall (1959), The theory of groups (Macmillan, New York).

P. Hall (1957), Nilpotent groups (Canad. Math. Cong. Univ. Alberta.) (Queen Mary College Math. Notes, (1970)).

M. R. Jones (1972), 'Multiplicators of p-groups', Math. Z. 127, 165-166.

C. R. Leedham-Green and S. McKay (1976), 'Baer-invariants, isologism, varietal laws and homology, Acta Math. 137, 99-150.

M. R. R. Moghaddam (1975), A varietal generalisation of the Schur multiplicator (Ph.D. Thesis, University of London).

M. R. R. Moghaddam (1979), 'The Baer-invariant of a direct product', Arch. Math. (Basel) 33, 504-511.

H. Neumann (1967), Varieties of groups (Springer, Berlin).

D. J. S. Robinson (1972), Finiteness conditions and generalized soluble groups, Part 1 (Springer, Berlin).

I. Schur (1907), 'Untersuchungen über die Darstellung der endlichen Gruppen durch gebrochene lineare Subtitutionen', J. Reine Angew. Math. 132, 85-137.

R. F. Turner-Smith (1964), 'Marginal subgroup properties for outer commutator words,' Proc. London Math. Soc. 14, 321-341.

J. Wiegold (1965), 'Multiplicators and groups with finite central factor-group', Math. Z. 89, 345-347.

Department of Mathematics

Faculty of Science

University of Mashhad

Mashhad

Iran 